\title{
MULTI-MODE OPERATION OF THE ACCELERATOR RF CAVITY.
}

\author{
Daniel Wisnivesky ${ }^{\sharp}$ LNLS and IFGW Unicamp, Campinas, Brazil
}

\begin{abstract}
In this paper we discuss a new mode of operation and design criteria of the RF cavity of a circular accelerator. It is shown that, for a specified energy aperture, a room temperature RF cavity can operate with a significant reduction in wall heat dissipation if, together with the fundamental mode, high order modes of a properly design cavity are simultaneously excited.
\end{abstract}

\section{INTRODUCTION}

Room temperature RF cavities used in circular accelerators are low efficient components. A large fraction of the RF power delivered to the cavity is dissipated in the cavity walls, while, in some cases, a relatively small part is effectively used to accelerate the beam. In a synchrotron accelerator the efficiency is typically of the order of 50\%. In general, several tens of $\mathrm{kW}$ are transformed into heat on the cavity walls in order to get the energy aperture and accelerating field needed. To control cavity detuning and to avoid undesirable high order mode (HOM) resonances, the cavity temperature is required to stay constant, which in general implies a large cooling system. As a consequence, the RF cavity design results in a compromise among conflicting requirements: ultra high vacuum, high heat dissipation, high electric fields and precise temperature and frequency control. It is clear that much could be gained if the system would be able to operate with a substantial reduction of the heat load in the cavity.

The spectrum of HOM is another aspect that deserves careful attention in the design and operation of the RF cavity. Special effort is devoted to either provide for methods that would damp the Q of the HOM, or temperature control the resonant frequencies so as to avoid undesirable coincidences with the revolution frequency or any of its multiples. In general, HOM are seen as an inconvenient consequence of the discrete spectrum of the system.

In this paper we want to discuss a different mode of operation of the RF cavity: multi-mode excitation. The cavity is designed to resonate at specifically chosen frequencies. In this cavity, several HOM modes are simultaneously excited and carefully combined in such a way that they provide for the necessary longitudinal acceleration of the beam and the required energy aperture

\footnotetext{
"Email: daniel@lnls.br
}

with a significantly reduction of the total power dissipated in walls.

An available growing number of high frequency, high power amplifiers, together with a large experience in the design of complex cavities makes it tenable to discuss multi-mode excitation as a practical possibility.

\section{EFFECTIVE FIELD AND ENERGY APERTURE OF HOM}

We consider a cavity that is simultaneously excited with different transverse magnetic modes. The longitudinal component of the TMonp mode electric field along the axis of the cavity is given by the equation:

$$
E=E_{n p} \cos \left(\frac{p \pi z}{d}\right) \sin \left(\frac{2 \pi t}{T_{p}}\right)
$$

where $\mathbf{f p}=\mathbf{1} / \mathbf{T p}$ is the frequency of the mode. The time needed for a particle to transverse the cavity is

$$
T_{0}=d / \beta_{0} c \text {. }
$$

with $\mathbf{d}$, the axial length of the cavity.

The effective electric field sensed by a charged particle going through the cavity is given by the expression

$$
E_{\text {eff }}=\frac{E_{n p}}{d} \int_{z\left(-\frac{T_{0}}{2}+t\right)}^{z\left(\frac{T_{0}}{2}+t\right)} \cos \left(\frac{p \pi z}{d}\right) \sin \left(\frac{2 \pi t}{T_{p}}\right) d z
$$

computed along the particle trajectory, $z=\beta_{0} c t$.

Evaluation of equation (2) results in an expression for the effective $E$ field of the form:

$$
E_{e f f}=E_{n p}\left[\frac{\sin \left(\frac{\pi T_{0}}{T_{p}}-p \frac{\pi}{2}\right) \sin \left(\frac{2 \pi}{T_{p}}-\frac{p \pi}{T_{0}}\right) t}{\left(\frac{2 \pi T_{0}}{T_{p}}-p \pi\right)}+\frac{\sin \left(\frac{\pi T_{0}}{T_{p}}+p \frac{\pi}{2}\right) \sin \left(\frac{2 \pi}{T_{p}}+\frac{p \pi}{T_{0}}\right) t}{\left(\frac{2 \pi T_{0}}{T_{p}}+p \pi\right)}\right]
$$

In general, neither one of the oscillatory terms in equation (3) will be coherent with the fundamental mode. Nevertheless, by proper design of the cavity, it is possible for the frequency of each TMonp mode to satisfy the relationship:

$$
\frac{2}{T_{p}}-\frac{p}{T_{0}}=\frac{2}{T}
$$

where $\mathbf{f}=\mathbf{1 / T}$ is the frequency of the fundamental mode. In that case, the effective field contribution of TMonp is 
$E_{\text {eff }}=\frac{E_{n p}}{2} \frac{\sin \left(\pi \frac{T_{0}}{T}\right)}{\pi \frac{T_{0}}{T}}\left[\sin \frac{2 \pi t}{T}+(-1)^{p} \frac{1}{\left(1+\frac{p T}{T_{0}}\right)} \sin \left(1+\frac{p T}{T_{0}}\right) \frac{2 \pi t}{T}\right]$

with a corresponding mode frequency given by

$$
f_{p}=f+p / 2 T_{0}
$$

Equation (5) indicates that, for $\mathrm{p}>0$, the TMonp mode has 2 contributions to the effective $E$ field:

- an electric field with the same frequency as the fundamental mode and $1 / 2$ its effective amplitude,

- a component with a larger frequency $f_{\text {equiv }}^{p}=f+p / T_{0}$, and an amplitude reduced by a factor $\mathrm{T} 0 /(\mathrm{T} 0+\mathrm{pT})$.

The corresponding contribution to the energy acceptance [1] can be obtained, for large over voltage from the integral

$$
A_{p}=\int_{0}^{T / 2} E_{e f f}(t) d t
$$

which reduces to

$$
A_{p}=E_{n p} \frac{\sin \left(\pi \frac{T_{0}}{T}\right)}{\pi \frac{T_{0}}{T}} \frac{T}{\pi}\left[\frac{1}{2}+\frac{(-1)^{p}}{4} \frac{1+\cos \left(\frac{p T}{T_{0}}\right)}{\left(1+\frac{p T}{T_{0}}\right)^{2}}\right]
$$

The first term in equation (7) shows that all p-modes make an equal contribution to the energy aperture of $1 / 2$ the value of the contribution of the fundamental mode. The second term, corresponding to the higher frequency excitation of the cavity, adds or subtracts a small correction to the value of the energy acceptance. We will neglect this contribution in the following discussion.

If several modes are simultaneously excited, the electric field of each one could be smaller than the amplitude of the field in case of single mode excitation. This indicates that, multi-mode excitation of the cavity makes possible to reduce the total power dissipation while keeping the same energy aperture. Something similar to what occurs when several cavities are used in substitution of a single cavity. If we assume that the cavity is exited with s modes with frequencies $\mathrm{fp}=\mathrm{f}+\mathrm{p} / 2 \mathrm{~T}$ and amplitudes Ep $(\mathrm{p}=0,1, \ldots \mathrm{s})$, the energy acceptance is proportional to

$$
\mathrm{E}_{a c c} \approx E_{0}+\frac{1}{2} E_{1}+\ldots+\frac{1}{2} E_{s}
$$

and the power dissipation is proportional to

$$
P \approx \frac{E_{0}^{2}}{R_{0}}+\frac{E_{1}^{2}}{R_{1}}+\ldots+\frac{E_{s}^{2}}{R_{s}}
$$

where $\mathrm{R} 0, \mathrm{R} 1, \ldots, \mathrm{Rs}$ are the corresponding shunt impedance.

Minimum power in the cavity is obtained if:

$$
\begin{aligned}
& E_{1}=\frac{R_{1}}{2 R_{0}} E_{0} \\
& \cdots \\
& E_{s}=\frac{R s}{2 R_{0}} E_{0}
\end{aligned}
$$

In this case, for the same energy acceptance, the power dissipated in the cavity in the multi mode case is given, in terms of the corresponding power dissipation for a single mode, by the equation

$$
P=\frac{1}{1+\frac{R_{1}}{4 R_{0}}+\ldots+\frac{R s}{4 R_{0}}} P_{0}
$$

We can make an estimate of the multi-mode power in the cavity if we take the case when all shunt impedance are approximately equal. Table I gives the multi-mode power dissipated in the cavity in terms of the single mode power, considering different number of modes. It also shows the fraction of the total power in the cavity delivered by each generator.

\begin{tabular}{|c|l|l|l|l|l|}
\hline $\begin{array}{l}\text { Number } \\
\text { of } \\
\text { modes } \\
\text { in the } \\
\text { cavity }\end{array}$ & $\begin{array}{l}\text { Total } \\
\text { multi- } \\
\text { mode } \\
\text { power } \\
\text { (P) } \\
\text { relative } \\
\text { to single } \\
\text { mode } \\
\text { power }\end{array}$ & $\begin{array}{l}\text { Relat. } \\
\text { power } \\
\text { in } \\
\text { mode } \\
\text { f0 }\end{array}$ & $\begin{array}{l}\text { Relat. } \\
\text { power } \\
\text { in } \\
\text { mode } \\
\text { f1 }\end{array}$ & $\begin{array}{l}\text { Relat. } \\
\text { power } \\
\text { in } \\
\text { mode }\end{array}$ & $\begin{array}{l}\text { Relat. } \\
\text { f2 } \\
\text { power } \\
\text { in } \\
\text { mode } \\
\text { f3 } \\
\text { P3/P }\end{array}$ \\
\hline 1 & $100 \%$ & 1 & - & - & - \\
\hline 2 & $80 \%$ & 0.8 & 0.2 & - & - \\
\hline 3 & $66 \%$ & 0.66 & 0.16 & 0.16 & - \\
\hline 4 & $57 \%$ & 0.57 & 014 & 0.14 & 0.14 \\
\hline
\end{tabular}

Table I: multi-mode power relative to single mode power for different numbers of modes in the cavity. 


\section{DISCUSSION OF A CAVITY DESIGN}

As an example, we have discussed the design of a simple cavity for 2 modes operation. The main design parameters are:

Number of modes: 2 ( $\mathrm{s}=1)$.

Fundamental mode: TM010

Frequency: $300 \mathrm{MHz}$.

Cavity length d: $500 \mathrm{~mm}$.

Second mode: TM011

Frequency: $600 \mathrm{MHz}$, in agreement with equation (6)

The simulation was made using Superfish [2] and adjusting the cavity shape so as to obtain a large $Q$ and shunt impedance for each mode. Figure 1 shows the general boundary of the cavity

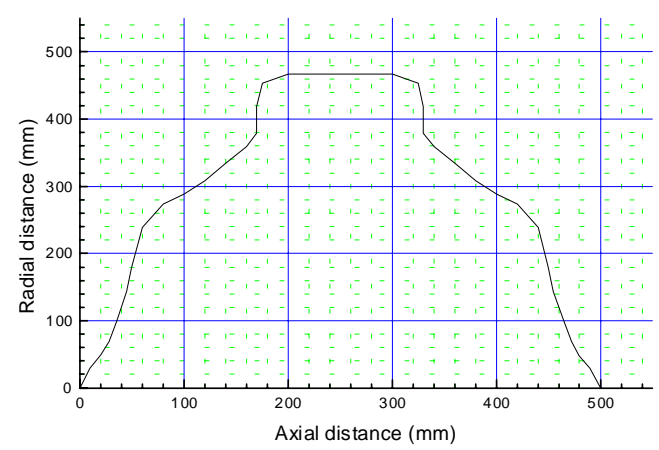

Figure 1 . General picture of the $300 / 600 \mathrm{MHz}$ cavity

The main results obtained by Superfish are summed up in table II.

\begin{tabular}{|l|l|l|l|}
\hline $\begin{array}{l}\text { Cavity } \\
\text { mode }\end{array}$ & Frequency & $\begin{array}{l}\text { Shunt } \\
\text { Impedance }\end{array}$ & Q \\
\hline TM010 & $300 \mathrm{MHz}$ & $14 \mathrm{M} \Omega$ & 45,000 \\
\hline TM011 & $600 \mathrm{MHz}$ & $11.5 \mathrm{M} \Omega$ & 60,800 \\
\hline
\end{tabular}

Table II: Superfish parameters for the modes TM010 and TM011.

As a working example we can compute the power requirements of this cavity when 2 modes are excited as compared to the single mode operation.

According to equation (9), for every $10 \mathrm{~kW}$ dissipated in the $300 \mathrm{MHz}$ resonance in single mode operation we get:

\begin{tabular}{|c|c|c|}
\hline & $\begin{array}{c}\text { Power } \\
\text { dissipated in } \\
300 \mathrm{MHz}\end{array}$ & $\begin{array}{c}\text { Power } \\
\text { dissipated in } \\
600 \mathrm{MHz}\end{array}$ \\
\hline $\begin{array}{c}\text { For every 10 } \\
\text { kW single } \\
\text { mode } \\
\text { dissipation }\end{array}$ & $5.7 \mathrm{~kW}$ & $2.6 \mathrm{~kW}$ \\
\hline
\end{tabular}

Table III: Power dissipation in the modes TM010 and TM011.
If the cavity is adjusted to have in addition a TMon2 resonance at $900 \mathrm{MHz}$ we get (assuming similar shunt impedance for TM011 and TM012) a power dissipation distributed in each one of the 3 modes according to table $\mathrm{IV}$, for every $10 \mathrm{~kW}$ single mode dissipation:

\begin{tabular}{|c|c|c|c|}
\hline & $\begin{array}{c}\text { Power } \\
\text { dissipated } \\
\text { in } 300 \\
\mathrm{MHz}\end{array}$ & $\begin{array}{c}\text { Power } \\
\text { dissipated } \\
\text { in } 600 \\
\mathrm{MHz}\end{array}$ & $\begin{array}{c}\text { Power } \\
\text { dissipated } \\
\text { in } 900 \\
\mathrm{MHz}\end{array}$ \\
\hline $\begin{array}{c}\text { For every } \\
10 \mathrm{~kW}\end{array}$ & $5.0 \mathrm{~kW}$ & $1.1 \mathrm{~kW}$ & $1.1 \mathrm{~kW}$ \\
\hline
\end{tabular}

Table IV: Power dissipation distributed in 3 modes.

\section{CONCLUSIONS}

We have discussed the power dissipation in the accelerator RF cavity when several resonant modes are exited. We have shown that, with an adequate design of the cavity, these modes make a significant contribution to the energy aperture of the ring. Thus, the amplitude of each mode and the power dissipation are smaller than in the normal single-mode excitation of the cavity. The reduction in power dissipation increases with the number of modes. The effect is similar to the one produced by augmenting the number of cavities in the ring: the field amplitude in each cavity is smaller and the total power is reduced. In the present case, no additional cavities are required. Instead a more complex multi frequency transmitter is involved. The growing number of high frequency, high power transmitters available in recent years makes multi-mode operation of the accelerator RF system a practical possibility.

\section{REFERENCES}

[1]/ M. Sands. The physics of electron storage rings. Int. Sch. of Physics "Enrico Fermi" Academic Press 1971, p 342.

[2]/ Superfish Code. PC version distributed by LAACG. Laacg.lanl.gov 